\title{
Low-dimensional weakly interacting Bose gases: Nonuniversal equations of state
}

\author{
G. E. Astrakharchik, ${ }^{1}$ J. Boronat, ${ }^{1}$ I. L. Kurbakov, ${ }^{2}$ Yu. E. Lozovik, ${ }^{2}$ and F. Mazzanti ${ }^{1}$ \\ ${ }^{1}$ Departament de Física i Enginyeria Nuclear, Campus Nord B4-B5, Universitat Politècnica de Catalunya, E-08034 Barcelona, Spain \\ ${ }^{2}$ Institute of Spectroscopy, RU-142190 Troitsk, Moscow Region, Russia
}

(Received 30 July 2009; revised manuscript received 9 October 2009; published 19 January 2010)

\begin{abstract}
The zero-temperature equation of state is analyzed in low-dimensional bosonic systems. We propose to use the concept of energy-dependent $s$-wave scattering length for obtaining estimations of nonuniversal terms in the energy expansion. We test this approach by making a comparison to exactly solvable one-dimensional problems and find that the generated terms have the correct structure. The applicability to two-dimensional systems is analyzed by comparing with results of Monte Carlo simulations. The prediction for the nonuniversal behavior is qualitatively correct and the densities, at which the deviations from the universal equation of state become visible, are estimated properly. Finally, the possibility of observing the nonuniversal terms in experiments with trapped gases is also discussed.
\end{abstract}

DOI: 10.1103/PhysRevA.81.013612

PACS number(s): 03.75.Hh, 51.30.+i, 34.50.Bw

\section{INTRODUCTION}

Understanding the properties of rarefied quantum systems is a fundamental question that has been addressed in a large number of works. This problem was extensively studied in the 1950s-1960s when significant development of mathematical formalism such as perturbative methods, Feynman diagrams, diagonalization techniques, etc. (see, for example, [1,2]) permitted researchers to obtain important results and attracted a lot of interest to dilute quantum systems. Some important results were also obtained in low-dimensional systems [3,4], which at that moment were rather mathematical toys with reduced applicability in the real world. The situation changed radically with the realization of Bose-Einstein condensation in dilute gases [5,6]. By having an excellent experimental control over the geometry of the cloud it was possible to create essentially pure quantum gases in the dilute regime and to probe the system properties. The experimental advances in the field with the realization of very anisotropic traps stimulated further the interest in dilute low dimensional gases (see, for example, Refs. [7-12]).

In the ultradilute limit the interparticle potential can be described by one parameter, namely the $s$-wave scattering length $a$, and the ground state properties of a gas are governed by the gas parameter $n a^{D}$, where $n$ is the particle density and $D$ stands for the dimensionality. As the density is increased, details of the interaction potential become important. Such a nonuniversal regime has been thoroughly studied in three-dimensional (3D) geometries, where the universal terms are known $[13,14]$. Low-energy corrections coming from the specific interaction potential can be described by the effective range $r_{0}$. Corrections to the ground-state energy, excitation spectrum, and the condensate fraction can be obtained (see, for example, Refs. [15,16] and more recent works [17,18]). Also, it was shown that for two-body problems the inclusion of an energy-dependent pseudopotential improves significantly upon the use of an energy-independent pseudopotential [19]. Also, the concept of a momentum dependent scattering length is very useful for estimation of the interaction for a Rydberg atom because it allows the effect of the Coulomb potential of nucleus to be taken into account (see, for example, Ref. [20]).
Unfortunately, much less is known in low-dimensional systems. Only recently has an analytical expression for the equation of state of a two-dimensional (2D) Bose gas in the low density regime $n a^{2} \lesssim 10^{-3}$ been correctly derived [21-23] and checked numerically [24]. In these works the interaction potential is described only by the $s$-wave scattering length $a$. So far, no analytical expression for the potential-dependent equation of state is known. The main goal of the present study is to obtain nonuniversal corrections to the universal equation of state of low-dimensional systems.

We propose to substitute in the universal equation of state the $s$-wave scattering length by the energy-dependent one in order to generate the leading potential-dependent energy terms. Our physical hypothesis is that by improving the description at a two-body level we also obtain the dominant corrections to the mean-field many-body energy. Still, we cannot prove that the applied techniques will work for systems in general.

The rest of the article is organized as follows. In Sec. II we discuss the origins of the universal behavior and study the two-body scattering problem and propose a simple way to obtain nonuniversal corrections. In Sec. III the equation of state of some exactly solvable one-dimensional (1D) models are analyzed. Some properties of 2D systems are addressed in Sec. IV. We start with an overview of the literature in Sec. IV A. In Sec. IV B we discuss the expansion of the universal equation of state and provide some physical insight on the origins of the beyond mean-field (BMF) terms. The knowledge of the expansion of the universal equation of state permits us to investigate the nonuniversal equation of state as it comes from the method proposed in Sec. II and to confront that with numerical results. Section IV C is devoted to the study of nonuniversal effects in the $s$-wave scattering problem and in the many-body equation of state. In Sec. V, we discuss the possibility of experimental observation of nonuniversal effects in trapped, cold gases. The feasibility of reaching an ultradilute $2 \mathrm{D}$ regime is also discussed. Finally, the main conclusions are drawn in Sec. VI.

\section{UNIVERSAL AND NONUNIVERSAL TERMS}

In dilute systems the probability of three-body collisions is highly reduced, leaving two-body scattering as the most 
important physical process. In this process two particles scatter each other with a relative momentum $k$. The two-body scattering problem is described by the Schrödinger equation

$$
-\frac{\hbar^{2}}{m} \Delta \psi(r)+V_{\mathrm{int}}(r) \psi(r)=\frac{\hbar^{2} k^{2}}{m} \psi(r) .
$$

If the interaction potential $V_{\text {int }}(r)$ is short-ranged, its exact shape is not important at low density and the relevant quantity of the scattering solution $\psi(r)$ is the phase $\delta(k)$ at distances larger than the range of the potential. For small scattering energies, the phase can be expanded in terms of the momentum $k$. In a $3 \mathrm{D}$ system this leads to

$$
k \cot \delta(k)=-\frac{1}{a_{0}}+\frac{1}{2} k^{2} r_{0}+\cdots,
$$

where $a_{0}$ is the $s$-wave scattering length and $r_{0}$ is the effective range. If the scattering momentum is very small the only relevant parameter is the $s$-wave scattering length $a_{0}$ and all potentials having the same value of $a_{0}$ will behave similarly. This limit is known as the universal regime. The relevant length scales are then $a_{0}$ and the interparticle distance. It is expected that the many-body ground-state energy can be expressed in terms of the gas parameter $n a_{0}^{D}$, where $D$ denotes the dimensionality of the problem.

For example, the low density energy per particle of a homogeneous, weakly-interacting Bose gas in 3D at zero temperature is given by

$$
\frac{E_{3 \mathrm{D}}}{N}=\frac{2 \pi \hbar^{2} n a_{0}}{m}\left(1+\frac{32}{15 \pi} \sqrt{16 \pi n a_{0}^{3}}+\cdots\right),
$$

with the leading term linear in the density being the mean-field Gross-Pitaevskii contribution [25] and quantum fluctuations contributing to the subleading $n^{3 / 2}$ Lee-Huang-Yang correction $[13,14]$. The next term scales like $n^{2}$, but it is no longer universal $[15,16]$ and depends on the explicit choice of the interaction potential.

It is possible to recast the definition Eq. (2) of the scattering length $a_{0}$ in a different form; namely, as the position of the node of the analytic continuation of the scattering solution from distances much larger than the range of the potential in the zero-energy scattering limit. Indeed, in 3D and in the limit of very low-scattering energy, the phase reads $\delta(k)=-k a_{0}$ and the scattering solution becomes $\sin (k r+\delta) / r \rightarrow k\left(r-a_{0}\right) / r$, which has a node at $r=a_{0}$. The advantage of this alternative definition is that it is well suited also for low-dimensional problems. An example how the $s$-wave scattering length changes with the type of the potential is shown in Fig. 1 for several characteristic interactions in 1D. The figure shows the asymptotic continuation of the zero-energy scattering solution.

We generalize the definition of the $s$-wave scattering length to finite values of the scattering energy.

Definition 1. The generalized scattering length $a(k)$ is the position of the node of the analytical continuation of the large distance $r \rightarrow \infty$ two-body scattering solution $\psi(r)$ at the scattering energy $\hbar^{2} k^{2} / m$. If there are several nodes, the position of the closest node to $r=0$ has to be taken.

In this way the $s$-wave scattering length $a(k)$ depends on the scattering momentum and fulfills the condition $\lim _{k \rightarrow 0} a(k)=$ $a_{0}$. Some typical examples of the dependence on the moment $k$ of the incident particle are shown in Fig. 2. An abrupt

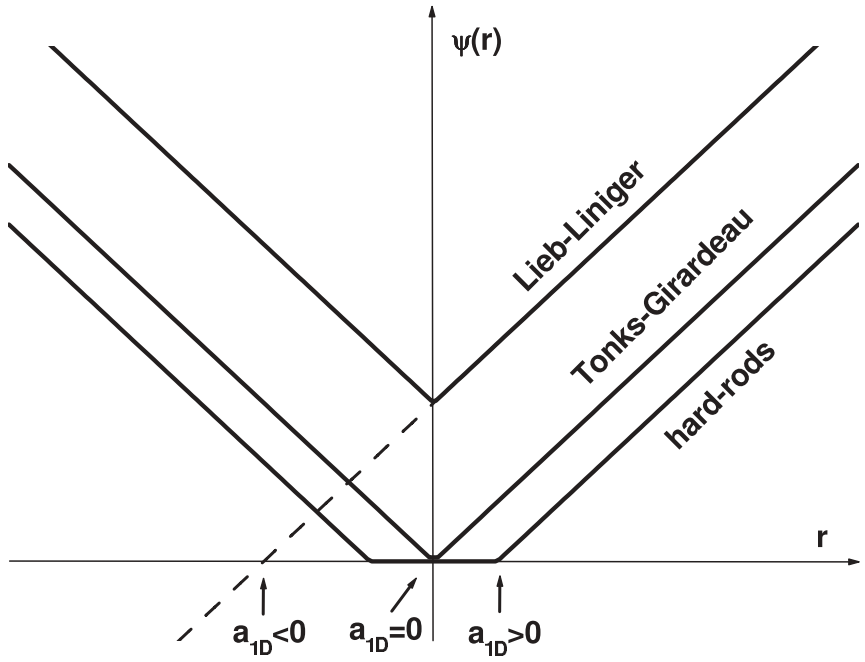

FIG. 1. Solid lines are the typical two-body scattering solutions $\psi(r)$ at zero energy for Lieb-Liniger (upper curve), Tonks-Girardeau (middle curve), and hard-rod (lower curve) Hamiltonians. The dashed lines are the analytic continuation of the scattering solution for the Lieb-Liniger model. Arrows indicate the positions of the nodes.

deviation of $a(k)$ from the zero-energy value $a_{0}$ at some characteristic scattering moment $k_{c}$ defines the region where the description in terms of $a_{0}$ can be applied. In order to estimate the characteristic value of the gas parameter $n_{c} a_{0}^{D}$ up to which the universal equation of state may be valid, it is sufficient to relate the typical scattering energy $\hbar^{2} k_{c}^{2} / m$ to the mean-field energy $g n / 2$. It is clear that the universal equation of state cannot be precise for densities larger than $n_{c}$, at the same time it is less evident that the corrections generated by using $a(k)$ will produce an accurate description of

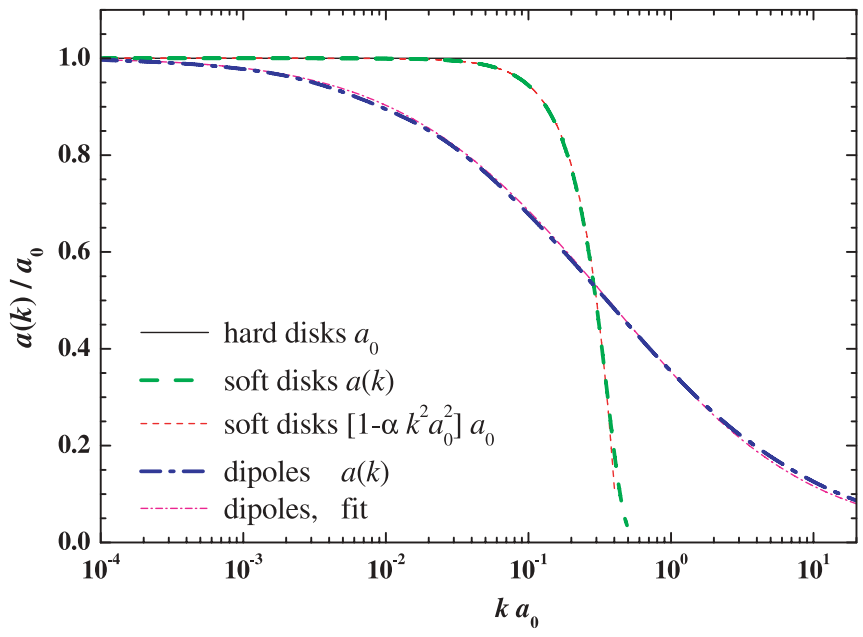

FIG. 2. (Color online) Finite-energy $s$-wave scattering length in $2 \mathrm{D}$ as a function of momentum $k$ of the incident particle for different interaction potentials. All quantities are measured in units of $a_{0}$. Solid line, hard disks $a(k)=a_{0}$; dashed lines, soft disks: thick line, numerical solution as the node of (C5), thin line, analytical expansion $a(k) / a_{0}=1-5.53454 k^{2} a_{0}^{2}$ as comes from (C9) using the parameters of soft disks taken from Ref. [51]; dash-dotted lines, repulsive dipoles $1 / r^{3}$ interaction: thick line, numerical solution, thin line, fit to Eq. (17). 
the energy. We will show in the next sections that the inclusion of the finite-momentum corrections improves the description of the energy and allows us to correctly estimate the term of the expansion where the nonuniversal behavior appears.

At this point it is important to understand the relationship between the effective range and the $s$-wave scattering length in the description of nonuniversal effects. The effective-range theory is well established in 3D systems (see, for example, textbook [1]). The effective range is then defined from the expansion of the phase shift in terms of the scattering momentum, see Eq. (2). The constant term defines the $s$-wave scattering length $a_{0}$, and the effective range $r_{0}$ corresponds to taking into account the dependence on $k^{2}$. Instead, the energy-dependent $s$-wave scattering length $a(k)$ includes in addition all higher order momenta (i.e., $k^{2}, k^{4}, k^{6}, \ldots$ ). More importantly, the concept of $a(k)$ can be applied to low-dimensional systems, where the nonuniversal terms in the equation of state are not generally known. In our approach it is enough to know the dependence on $a_{0}$ of the universal equation of state and the nonuniversal terms will be automatically generated.

\section{ONE-DIMENSIONAL SYSTEMS}

One peculiarity of the 1D world is that several many-body models can be solved exactly (with short-range $[3,4]$ and longrange [26] interactions), in the sense that the exact ground state can be written either explicitly $[3,26]$ or can be easily obtained as the solution of a system of integral equations [4]. This allows us to test the proposed approach of using an energy-dependent scattering length by comparing to the exactly known results.

The ground-state energy of a Bose gas with a repulsive $\delta$-pseudopotential interaction (Lieb-Liniger model) can be obtained by solving the Bethe ansatz equations. The expansion of the energy in the mean-field regime [4] has a structure similar to that of the $3 \mathrm{D}$ case Eq. (3):

$$
\frac{E_{1 \mathrm{D}}}{N}=\frac{1}{2} g_{1 \mathrm{D}} n_{1 \mathrm{D}}\left(1-\frac{4 \sqrt{2}}{3 \pi}\left(n\left|a_{1 \mathrm{D}}\right|\right)^{-1 / 2}+\cdots\right),
$$

where $g_{1 \mathrm{D}}=-2 \hbar^{2} /\left(m a_{1 \mathrm{D}}\right)>0$ is the $1 \mathrm{D}$ coupling constant. Indeed, the leading term in Eq. (4) is the same as it would come out from the mean-field Gross-Pitaevskii equation, while the subleading term is the same as obtained from Bogoliubov theory. In passing, we note that such a coincidence is not obvious a priori, as both the Gross-Pitaevskii and Bogoliubov theories assume that all or a large fraction of particles are in the condensate. Instead, strictly speaking, Bose-Einstein condensation in homogeneous 1D system is absent [27].

The reason why the theories based on the presence of a Bose condensate produce correct results for energetic properties can be understood by following the similar arguments used in the renormalization group approach (see, e.g., Ref. [28]). The main contribution to the energy comes from short distances. At short distances the phase coherence may be present even in the absence of Bose-Einstein condensation. Therefore, on this length scale it is possible to apply the perturbative theories that are based on the assumptions of a macroscopic occupation of the condensate. Coherence at finite distances larger than the interparticle distance is sufficient for MF and Bogoliubov theories to yield the correct ground-state energy. In particular, such theories successfully describe 1D systems at zero temperature (such as a Lieb-Liniger gas in the regime of weak correlations) and 2D dilute Bose gases at finite temperature, none of which has true Bose-Einstein condensation. A mathematical way to resolve the paradox and to prove the validity of the Bogoliubov result in 1D systems is to use space discretization and to introduce the concept of a quasi-condensate [29].

Contrary to $3 \mathrm{D}$ and $2 \mathrm{D}$ systems, here the mean-field regime means high densities $n_{1 \mathrm{D}} a_{1 \mathrm{D}} \gg 1$. This precludes us from using the concept of energy-dependent scattering length in the MF regime, as the energy of an incident particle would be huge [see Eq. (4)], as would the deviations of $a(k)$ from $a_{0}$. Thus, the mean-field regime is no longer universal (contrary to what happens in 3D and 2D systems), as the energy and correlation functions are very different for the $\delta$-pseudopotential [4,30,31], the Calogero-Sutherland $1 / z^{2}$ potential [26,32], and the dipolar $1 /|z|^{3}$ interaction [33]. Instead, in the regime of strong quantum correlations, $n\left|a_{1 \mathrm{D}}\right| \ll 1$, the energy and correlation functions of all those models (essentially, for any repulsive interaction potential) approach the same universal limit referred as the Tonks-Girardeau [3] regime (see also Fig. 1).

A peculiar feature of the $1 \mathrm{D}$ world is that the dilute regime, $n_{1 \mathrm{D}}\left|a_{1 \mathrm{D}}\right| \ll 1$, corresponds not to a mean-field limit, but rather to a regime where quantum fluctuations are dominant. The energy in this limit is given by the energy of an ideal Fermi gas $E / N=\pi^{2} \hbar^{2} n_{1 \mathrm{D}}^{2} /(6 m)$ and the wave function of strongly interacting bosons can be mapped onto a wave function of noninteracting fermions $[3,34,35]$. For instance, the energy of a gas of hard rods of size $a_{1 \mathrm{D}}>0$ is obtained from the energy of an ideal Fermi gas by taking into account the excluded volume [3]: $n_{1 \mathrm{D}} \rightarrow N /\left(L-N a_{1 \mathrm{D}}\right)$. Expanding this expression in terms of the $1 \mathrm{D}$ gas parameter $\tilde{n}=n_{1 \mathrm{D}} a_{1 \mathrm{D}}$ at small densities $n \ll 1$ one gets [36]

$$
\frac{E_{H R}}{N}=\frac{\pi^{2} \hbar^{2} n_{1 \mathrm{D}}^{2}}{6 m}\left(1+2 \tilde{n}+3 \tilde{n}^{2}+4 \tilde{n}^{3}+\cdots\right) .
$$

Starting from the expansion for a 1D analog for hard spheres, Eq. (5), we will calculate the first nonuniversal corrections for a different potential. We chose a $\delta$-pseudopotential, as its exact ground-state energy is known and thus we can test our approach. The solution of the scattering problem of Eq. (1) with $V_{\text {int }}(r)=g_{1 \mathrm{D}} \delta(r)$ can be readily written as $\psi(r) \propto \sin \left(k|r|-\arctan k a_{1 \mathrm{D}}\right)$. The energy-dependent $s$ wave scattering length can be explicitly expressed as a function of the momentum and the leading correction to $a_{0}$ is quadratic in momentum:

$$
a(k)=\frac{\arctan k a_{1 \mathrm{D}}}{k}=a_{1 \mathrm{D}}-\frac{1}{3} k^{2} a_{1 \mathrm{D}}^{3}+\cdots .
$$

The substitution of Eq. (6) into Eq. (5) for a characteristic value of the energy $\hbar^{2} k^{2} / m \propto \pi^{2} \hbar^{2} n_{1 \mathrm{D}}^{2} /(6 m)$ allows us to estimate the first correction due to nonuniversality:

$$
\frac{E_{L L}^{\mathrm{appr} .}}{N}=\frac{\pi^{2} \hbar^{2} n_{1 \mathrm{D}}^{2}}{6 m}\left[1+2 \tilde{n}+3 \tilde{n}^{2}+\left(4-\frac{\pi^{2}}{9}\right) \tilde{n}^{3}+\cdots\right] .
$$

A possible concern about the validity of the obtained result is that expansion Eq. (5) is done for $n_{1 \mathrm{D}} a_{1 \mathrm{D}}>0$, while expansion 
Eq. (7) is used to describe a region where $n_{1 \mathrm{D}} a_{1 \mathrm{D}}<0$, with a different sign of the $s$-wave scattering length. We argue that the universal equation of state is smooth as a function of the $1 \mathrm{D}$ gas parameter $n_{1 \mathrm{D}} a_{1 \mathrm{D}}$. This is supported by the apparent similarities between the hard-rod gas and the gaslike state of the attractive $\delta$ pseudopotential ("super-Tonks-Girardeau" system) [37]. We also note that the Bethe ansatz solution for two-component attractive and repulsive fermions is continuous (compare results of Refs. [38-40]).

Equation (7) can be compared to the exact predictions for the Lieb-Liniger model based on the Bethe ansatz technique. The exact result can be obtained by solving the integral equations recursively (details are given in Appendix A) and reads

$$
\frac{E_{L L}}{N}=\frac{\pi^{2} \hbar^{2} n_{1 \mathrm{D}}^{2}}{6 m}\left[1+2 \tilde{n}+3 \tilde{n}^{2}+\left(4-\frac{14 \pi^{2}}{15}\right) \tilde{n}^{3}+\cdots\right] .
$$

By comparing the exact results for the hard-rod gas [Eq. (5)] with the exact results for the $\delta$-pseudopotential gas [Eq. (8)] and the approximate result [Eq. (7)] obtained by the proposed method we conclude that:

(i) The order of the expansion in which the $\delta$-pseudopotential and hard-rod energies differ is predicted correctly.

(ii) The expansion Eq. (8) contains the same rational terms as the expansion Eq. (5), while in addition it has irrational terms (here multiples of $\pi^{2}$ ). The use of an energy-dependent scattering length permits one to guess correctly the structure of the potential-dependent correction.

We find that the first three terms of the expansion are the same for the potentials considered. The physical meaning of such terms is that particles behave as if they were ideal fermions in the box of size $L-N a_{1 \mathrm{D}}$. Indeed, this interpretation explicitly applies to the hard-rod gas, where the excluded volume correction is negative, as $a_{1 \mathrm{D}}>0$. For a negative scattering length the "excluded volume" correction changes its sign and becomes positive: $L \rightarrow L+N\left|a_{1 \mathrm{D}}\right|$. In Fig. 1 we present the characteristic behavior of the one-body scattering solution at low energy for three short-ranged potentials. The Tonks-Girardeau potential corresponds to zero-range, infinitely strong repulsion. This places the node of the wave function at the origin and, according to Definition 1, the value of the $s$-wave scattering length is zero: $a_{1 \mathrm{D}}=0$. For a hard-rod interaction potential the position of the first node is positive and thus $a_{1 \mathrm{D}}>0$. The slope of $\psi(r)$ is determined by the scattering momentum [refer to Eq. (6) and the discussion above it], so for a similar scattering energy the only relevant difference in the wave function corresponding to different interactions is just a shift in abscissas. Thus, two Tonks-Girardeau particles separated by a distance $r$ and two hard-rod particles separated by a distance $r-a_{1 \mathrm{D}}$ "feel" each other in the same way. The only differences appear at very small distances of the order $r \approx a_{1 \mathrm{D}}$. In a similar way the scattering solution for a Lieb-Liniger Hamiltonian can be "adjusted" to match the Tonks-Girardeau solution by the change $r \rightarrow r-a_{1 \mathrm{D}}$ (mind that $a_{1 \mathrm{D}}<0$ in this case). This makes it natural that the "excluded volume" correction is encountered for different potentials, and it can change its sign. This was first noted in Ref. [41].

\section{TWO-DIMENSIONAL SYSTEMS}

\section{A. Overview of the equation of state}

A series of our previous works has been devoted to the study of the equation of state of dilute $2 \mathrm{D}$ Bose gases [24,42-47]. A number of interaction potentials (dipolar, hard disks, etc.) were considered in a wide range of densities. There, it was demonstrated that the dipoles crystalize at large densities. The density of the quantum phase transition turned out to be extremely large $n a_{0}^{2} \approx 2900$ [43,48,49]. This shows that the dipolar interaction potential is rather "soft" compared to hard-core potentials, which are expected to crystallize at values of the gas parameter that are smaller than unity (for example, $n a_{0}^{2}=0.33(2)$ in the case of hard disks [50]). The equation of state of a dilute gas was obtained both in the universal and nonuniversal regimes from Monte Carlo calculations for dipoles $[42,45]$ and hard and soft disks [51]. A peculiarity of the $2 \mathrm{D}$ systems is that the Gross-Pitaevskii equation has a limited applicability even in very dilute systems [44] due to the logarithmic dependence on the gas parameter rather than on powers of it, like in 3D and 1D systems. This means that it is extremely difficult to study numerically the universal equation of state. Densities as low as $n a^{2} \approx 10^{-100}$ had to be reached in calculations [24] in order to check numerically the low-density expansion of the universal equation of state. It turns out that in order to describe correctly the BMF effects, several terms have to be summed even at densities as low as $n a_{0}^{2} \approx 10^{-10}$ since the series comes out in terms of the slow converging logarithm function $\ln n a_{0}^{2}$, which at such densities is of the order of the next (constant) term. Historically, it turned out to be very difficult to obtain the correct expression for this term, see Ref. [24] for a summary of different results. Only recently has the correct expression been obtained [21-23,52].

Once the structure of the universal terms is well established, we are ready to test the concept of an energy-dependent, $s$ wave scattering length.

\section{B. Universal terms}

In this section we review the equation of state of $2 \mathrm{D}$ Bose gases in the universal regime; i.e., where the interaction potential can be described by one parameter, namely the $s$-wave scattering length, and all properties of the gas are fully defined by the gas parameter $n a^{2}$. Without going through a rigorous derivation of the equation of state, which would be an extremely tedious calculation, we provide some simple ideas that give insight into the relevant physics involved in the equation of state.

In a weakly interacting system of any dimensionality the leading contribution to the energy comes from mean-field theory. Assuming that the density is low enough, it does not matter what the exact shape of the short-range interaction potential is, and a simple $\delta$ pseudopotental can be used. In this way the real interaction potential can be replaced by a zero-range one, such that it imposes a correct zero-boundary 
condition to the scattering state

$$
V(r)=g \delta(r) \times(\text { regularization }) .
$$

The regularization operator is needed to make the $\delta$-function description compatible with a generic $1 / r$ (or $1 / \sqrt{r}$ ) divergence in a 3D (or 2D) geometry, although this is not important for our considerations. The substitution of Eq. (9) into the expression of the interaction energy written in first quantization simplifies the double integration $E=\frac{1}{2} \int d \mathbf{r}_{1} \int d \mathbf{r}_{2} \hat{\Psi}^{\dagger}\left(\mathbf{r}_{1}\right) \hat{\Psi}^{\dagger}\left(\mathbf{r}_{2}\right) V\left(\mid \mathbf{r}_{1}-\right.$ $\left.\mathbf{r}_{2} \mid\right) \hat{\Psi}\left(\mathbf{r}_{2}\right) \hat{\Psi}\left(\mathbf{r}_{2}\right)=(g / 2) \int d \mathbf{r} \hat{\Psi}^{\dagger}(\mathbf{r}) \hat{\Psi}^{\dagger}(\mathbf{r}) \hat{\Psi}(\mathbf{r}) \hat{\Psi}(\mathbf{r})$. Treating the field operator $\hat{\Psi}(\mathbf{r})$ as a classical field and substituting it with the particle density $\sqrt{n}$ one obtains the mean-field expression for the energy

$$
\frac{E}{N}=\frac{1}{2} g n
$$

It is easy to see from Eq. (9) that the coupling constant has dimensionality of $\left[E \times L^{D}\right]$ and it has to be expressed in terms of the parameters of the scattering problem, which are $\hbar, m$, and $a$. In the $3 \mathrm{D}$ case the considerations of units leads to a combination proportional to the $s$-wave scattering length $g_{3 \mathrm{D}} \propto \hbar^{2} a_{3 \mathrm{D}} / m$. Indeed, the exact expression is $g_{3 \mathrm{D}}=$ $4 \pi \hbar^{2} a_{3 \mathrm{D}} / m$. In a 1D system the correct units are obtained in a combination which is inversely proportional to the $s$-wave scattering length $g_{1 \mathrm{D}} \propto \hbar^{2} /\left(m a_{1 \mathrm{D}}\right)$. This agrees with the exact result $g_{1 \mathrm{D}}=-2 \hbar^{2} /\left(m a_{1 \mathrm{D}}\right)$. The $2 \mathrm{D}$ case is special in the sense that combinations having the proper units can be obtained without involving the $s$-wave scattering length $g_{2 \mathrm{D}} \propto \hbar^{2} / m$. The dependence on $a$ can come only in a combination with the scattering momentum $k$, which in a homogeneous system is related to the density. The exact result $[53,54]$ indeed has the structure $g_{2 \mathrm{D}}=4 \pi \hbar^{2} /\left(m\left|\ln n a_{2 \mathrm{D}}^{2}\right|\right)$, resulting in a mean-field expression of the total energy per particle [53]:

$$
\frac{E^{\mathrm{MF}}}{N}=\frac{2 \pi \hbar^{2} n}{m} \frac{1}{\left|\ln n a^{2}\right|} .
$$

The most important BMF terms were obtained by Popov [55] in 1972 (see also his book [28]). He obtained a recursive expression relating the chemical potential $\mu$ and the density $n$ for a given value of the inverse temperature $\beta$ :

$$
n=\frac{m \mu}{4 \pi \hbar^{2}}\left(\ln \frac{\varepsilon_{0}}{\mu}-1\right)-\int \frac{\hbar^{2} k^{2}}{2 m \varepsilon(k)} \frac{1}{e^{\beta \varepsilon(k)}-1} \frac{d^{2} k}{(2 \pi)^{2}},
$$

where $\varepsilon^{2}(k)=\left(\hbar^{2} k^{2} / 2 m\right)^{2}+\hbar^{2} k^{2} \mu / m$ is the Bogoliubov spectrum, and $\varepsilon_{0}$ is of the order of $\hbar^{2} / m r_{0}^{2}$, with $r_{0}$ the range of the interaction potential. We write the last relationship introducing an unknown coefficient of proportionality $C_{1}$ such that $\varepsilon_{0}=C_{1} \hbar^{2} / m a^{2}$.

At zero temperature quasiparticle excitations are absent and the expression simplifies. By solving Eq. (12) iteratively one obtains the following expression for the chemical potential:

$$
\mu^{p}=\frac{4 \pi \hbar^{2} n / m}{\left|\ln n a^{2}\right|+\ln \left|\ln n a^{2}\right|-\ln 4 \pi+\ln C_{1}-1 \cdots} .
$$

In 1978, Lozovik and Yudson used diagrammatic techniques to find a recursive relation which relates the chemical potential and the density [56]:

$$
\mu=\frac{4 \pi \hbar^{2} n_{0} / m}{\left|\ln \left(\mu m a_{0}^{2} / \hbar^{2}\right)\right|+O(1)} .
$$

Solving recursively Eq. (14) (see also [28] and Appendix B in [53]), one generates the first BMF term $\ln \left|\ln n a^{2}\right|$, some contributions to the second BMF term which is of order 1, and other analytically more involved terms containing logarithms of logarithms of $n a^{2}$.

Summarizing, one expects to find the following types of BMF corrections:

(i) a first BMF term of the form $\ln \left|\ln n a^{2}\right|$;

(ii) a second BMF constant term;

(iii) an additional contribution involving more complex combinations of logarithms of $n a^{2}$.

Historically, it took a long time to obtain correct BMF expansions at low densities (for a literature review refer to [24]). The double logarithm term can be obtained from the iterative relation Eq. (14) and it is present in the majority of theories. Unfortunately, this term alone is not sufficient to describe the universal regime and the calculation of all the contributions to the second BMF term was a challenging task. We note that the corresponding problem in 3D was solved in the 1950s [14] and the 1D problem in the 1960s [3,4].

The universal equation of state for the chemical potential should then read

$$
\mu=\frac{4 \pi \hbar^{2} n / m}{\left|\ln n a^{2}\right|+\ln \left|\ln n a^{2}\right|+C_{1}^{\mu}+\frac{\ln \left|\ln n a^{2}\right|+C_{2}^{\mu}}{\left|\ln n a^{2}\right|}+\cdots} .
$$

Notice that this expression is compatible both with Eq. (13) and the result of iterating Eq. (14) for $\mu$. The second BMF term was recently obtained analytically [21-23] as $C_{1}^{\mu}=-\ln \pi-$ $2 \gamma-1=-3.30 \ldots$ and its value was confirmed numerically in Ref. [24]. The subsequent constant was derived a short time ago in Ref. [52] with its value given by $C_{2}^{\mu}=-0.751$. In the following we will use a value obtained from a fit to Monte Carlo data $C_{2}^{\mu}=-0.3(1)$ [24].

The expansion of the energy per particle takes then a form similar to Eq. (15):

$$
\frac{E}{N}=\frac{2 \pi \hbar^{2} n / m}{\left|\ln n a^{2}\right|+\ln \left|\ln n a^{2}\right|+C_{1}^{E}+\frac{\ln \left|\ln n a^{2}\right|+C_{2}^{E}}{\left|\ln n a^{2}\right|}+\cdots},
$$

with the coefficients related as $C_{1}^{E}=C_{1}^{\mu}+1 / 2=-2.80 \ldots$ and $C_{2}^{E}=C_{2}^{\mu}+1 / 4$ (equals to $-0.05(10)$ from the numerical fit).

\section{Nonuniversal terms}

In Sec. II we have formulated our proposal for nonuniversal terms using an energy-dependent, $s$-wave scattering length. This allows us to generate nonuniversal terms in an energy expansion and also to understand analytically at which densities deviations from the universal law appear. This can be applied at densities for which the universal equation of state is known. As the reference equation of state we take Eq. (16). In this section we test our proposal for three different potentials, such as hard disks, soft disks, and dipoles. 


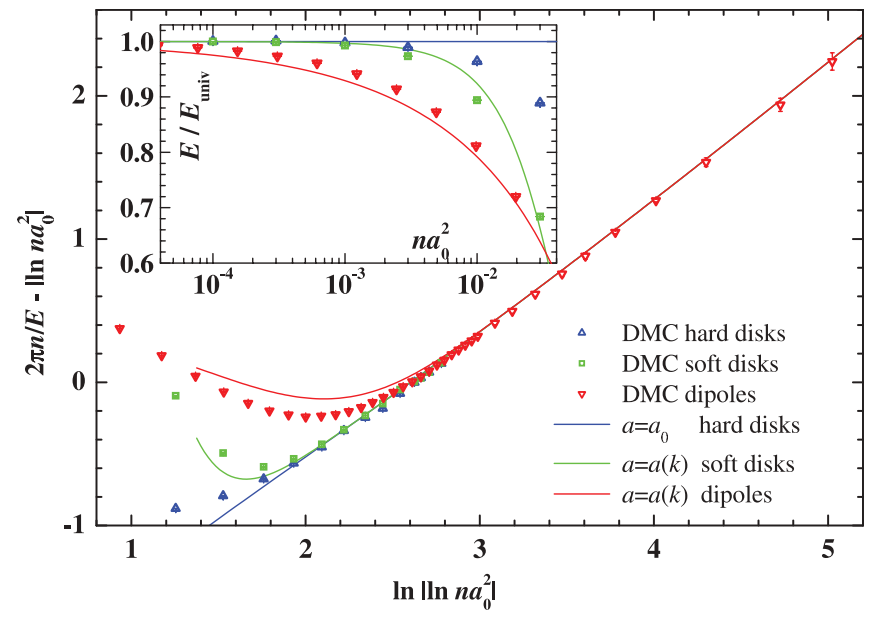

FIG. 3. (Color online) Energy per particle, analysis of nonuniversal BMF corrections. The main figure shows the BMF terms in the energy as a function of the double logarithm of the gas parameter. The upward-pointing triangles correspond to the hard disks, the squares to the soft disks, and the downward-pointing triangles to the dipoles. The curve for the hard disks is from Eq. (16), that for the soft disks is from Eq. (18), and that for the dipoles is from Eq. (16) with $a(k)$ as in Eq. (17). The inset shows the energy per particle $E / N$ in units of the "universal" equation of state, Eq. (16), as a function of the gas parameter $n a^{2}$.

In the case of hard disks, the interaction has only one length scale; namely, the size of the disk. As a result the energydependence is trivial: $a_{\mathrm{HD}}(k)=a_{0}$.
In the case of soft disks, corrections due to the finite scattering energy are important at typical densities $n a^{2} \gtrsim 10^{-3}$ [51]. The first correction due to the finite value of the scattering energy is quadratic in momentum, as shown in Appendix C. The explicit expression for $a(k)$ is given by formula (C9) and it reduces to $a_{\mathrm{SD}}(k)=a_{\mathrm{SD}}(0)\left(1-5.53454 k^{2}+\cdots\right)$ for the choice of soft-disk parameters as in Ref. [51].

The dipolar interaction potential decays slowly and deviations from the universal equation of state appear much earlier. In a very dilute system, $n a^{2} \lesssim 10^{-7}$, the dipoledipole scattering length is well approximated by its value at zero scattering momentum, $a_{\mathrm{dd}}(0)=e^{2 \gamma} r_{d}=3.17222 \cdots r_{d}$, where $r_{d}$ is a characteristic length scale for the dipole-dipole interaction potential [43]. We solve the $s$-wave scattering problem numerically and find that the following fit describes well the numerical data for the value of the $s$-wave scattering length at low energies:

$$
\frac{a_{\mathrm{dd}}\left(k r_{d}\right)}{a_{\mathrm{dd}}(0)}=e^{-\exp \left\{0.441082+0.31414 \ln k r_{d}-0.0275752 \ln ^{2} k r_{d}\right\}} \text {. }
$$

In order to find nonuniversal corrections to the energy, according to the proposed scheme, we substitute the gas parameter $n a_{0}^{2}$ in the universal expansion (16) with $n a^{2}(k)$. Within the level of accuracy of interest, it is sufficient to use the mean-field expression for the scattering momentum $k^{2} \propto 2 m E / N \hbar^{2}=4 \pi n /\left|\ln n a^{2}\right|$.

In the case of soft disks this leads to the substitution $\ln n a^{2} \rightarrow \ln n a_{0}^{2}+2 \ln \left(1-\alpha k^{2} a_{0}^{2}\right)$. The logarithm can be expanded as $\ln (1+\varepsilon)=\varepsilon+\cdots$, leading to nonuniversal corrections of the order of $2 \alpha k^{2} a_{0}^{2} \propto 8 \pi \alpha n a^{2} /\left|\ln n a^{2}\right|$. The resulting equation of state for soft disks then reads

$$
\frac{E}{N}=\frac{2 \pi \hbar^{2} n / m}{\left|\ln n a^{2}\right|+\ln \left|\ln n a^{2}\right|-\ln \pi-2 \gamma-1 / 2+\left[\ln \left|\ln n a^{2}\right|-\ln \pi-2 \gamma+2.0(1)+1 / 4+8 \pi \alpha n a^{2}\right] /\left|\ln n a^{2}\right|}
$$

The obtained analytical expressions for the equation of state are confronted with the results of Monte Carlo simulations. Figure 3 shows the BMF energy as a function of the double logarithm of the density for different interaction potentials (compare to Fig. 1 in Ref. [24]). As anticipated, the BMF terms have the most simple dependence for hard-disk potential (since the $s$-wave scattering length dependence is flat, see Fig. 2) and it is the best one described by the "universal" equation of state Eq. (16). The region where the description of the energy is universal shrinks in the case of soft disks and diminishes further for dipoles [compare to the dependence of the corresponding $a(k)$, Fig. 2]. We find that the analytical description we obtain for the nonuniversal behavior works rather well. In particular, the density at which deviations from the universal law start to be visible is correctly predicted by our approach. The analytical formula Eq. (18) provides not only a good qualitative description, but even the quantitative agreement is good. From Fig. 3 it might seem that the description is better for soft disks compared to dipoles, but in reality the description for both potentials is expected to have a similar level of accuracy. In order to check that we solved self-consistently the equations $E=E(n, a)$ and $a=a(E)$ (see Appendix B and Ref. [21]), thus obtaining a different expression, which have the same significative perturbation terms, but differ in higher order terms which are outside of the accuracy of our approach.

\section{DISCUSSION}

Recent progress in techniques of cooling and confinement permits the realization of extremely dilute gases in the regime of quantum degeneracy, thus providing a very advanced tool for studying the properties of weakly interacting gases. The $s$-wave scattering length can be controlled by using Feshbach resonances and can be set to, essentially, any desired value by choosing an appropriate magnetic field. Many features of the equation of state can be inferred from measuring energetic properties, such as release energies in time of flight experiments. Also, the size of the cloud and the density profile are related to the equation of state. At present, the most precise 
technique is the accurate measurement of the frequencies of collective oscillations. This method was successfully used to study BMF terms in the equation of state of two-component Fermi gases in the BCS-BEC crossover [57].

In previous sections we have investigated the properties of low-dimensional weakly interacting Bose gases as a function of the $1 \mathrm{D}$ and $2 \mathrm{D}$ gas parameters $n_{1 \mathrm{D}} a_{1 \mathrm{D}}$ and $n_{2 \mathrm{D}} a_{2 \mathrm{D}}^{2}$, respectively. The low-dimensional system can be realized in experiments by strongly squeezing the gas in one or two directions. Assuming that the trapping is harmonic with frequency $\omega$ the condition of being in a low-dimensional regime is that the oscillator levels should not be excited neither by the energy per particle nor by the temperature $E / N$, $k_{B} T \ll \hbar \omega$.

In a $1 \mathrm{D}$ system a relationship between the $3 \mathrm{D} a_{3 \mathrm{D}}$ and the $1 \mathrm{D}$ $a_{1 \mathrm{D}} s$-wave scattering lengths was found in Ref. [58] assuming harmonic radial confinement with oscillator length $a_{\text {ho }}$. The relationship has a resonant behavior when $a_{3 \mathrm{D}}$ is of the same order as $a_{\mathrm{ho}}$ because of the contribution of virtual excitations of the levels of transverse confinement. The 1D coupling constant $g_{1 \mathrm{D}}=-2 \hbar^{2} /\left(m a_{1 \mathrm{D}}\right)$ is expressed by Olshanii as [58]

$$
g_{1 \mathrm{D}}=\frac{2 \hbar^{2}}{m a_{\mathrm{ho}}^{2}} \frac{1}{1-1.0326 a_{3 \mathrm{D}} / a_{\mathrm{ho}}} .
$$

In particular, at the top of an Olshanii resonance, the 1D $s$-wave scattering vanishes and $a_{1 \mathrm{D}}=0$, making $g_{1 \mathrm{D}} \rightarrow \infty$. This corresponds to the Tonks-Girardeau limit. Close to the resonance $a_{1 \mathrm{D}}$ is small and expansions like Eqs. (5), (7) are applicable.

In a similar way to Eq. (19), the coupling constant in a quasi-2D system has a resonant structure [59]:

$$
g_{2 \mathrm{D}}=\frac{4 \pi \hbar^{2}}{m} \frac{1}{\left|\ln \left(2 \pi|\mu| m a_{\mathrm{ho}}^{2} / \hbar^{2}\right)\right|+\sqrt{2 \pi} a_{\mathrm{ho}} / a_{3 \mathrm{D}}},
$$

and describes a competition between a "purely 2D" logarithmic term and a mean-field Gross-Pitaevksii term $g_{\mathrm{Q} 2 \mathrm{D}}^{\mathrm{GP}}=$ $2 \sqrt{2 \pi}\left(\hbar^{2} / m\right) a_{3 \mathrm{D}} / a_{\mathrm{ho}}$, which can be obtained from the GrossPitaevskii energy functional assuming a Gaussian profile in the tight direction of the confinement. The results from Section IV apply to a purely $2 \mathrm{D}$ system, when the logarithmic term in Eq. (20) is dominant; i.e., when $a_{\mathrm{ho}} \ll a_{3 \mathrm{D}}$. We have explained that the mean-field (here, in a "purely" 2D sense) regime is achieved when the double logarithm of the $2 \mathrm{D}$ parameter is large, $\ln \left|\ln n a^{2}\right| \gg 1$, which leads to extremely rarefied densities such as $n a^{2} \ll 10^{-862}$. Fortunately, nature provides a way to get such small effective densities. Indeed, Eq. (20) can be rewritten introducing the second term of the denominator under the logarithm. The resulting expression can be interpreted in the sense of a "purely 2D" system, but with a rescaled effective density $n^{\star} \propto \exp \left(-\sqrt{2 \pi} a_{\mathrm{ho}} / a_{3 \mathrm{D}}\right) n$. Here the effect of large $a_{\mathrm{ho}} / a_{3 \mathrm{D}}$ ratios is exponentially amplified.

Expressions for the (quasi) low-dimensional coupling constants Eqs. (19), (20) were obtained from the analytic solution of the two-body scattering problems in the presence of a tight harmonic confinement [59]. The existence of 1D resonance in a many-body system was later confirmed in numerical simulations [60]. A similar 2D study is more involved as the expression of the coupling constant depends on the chemical potential and we are not aware of such studies.

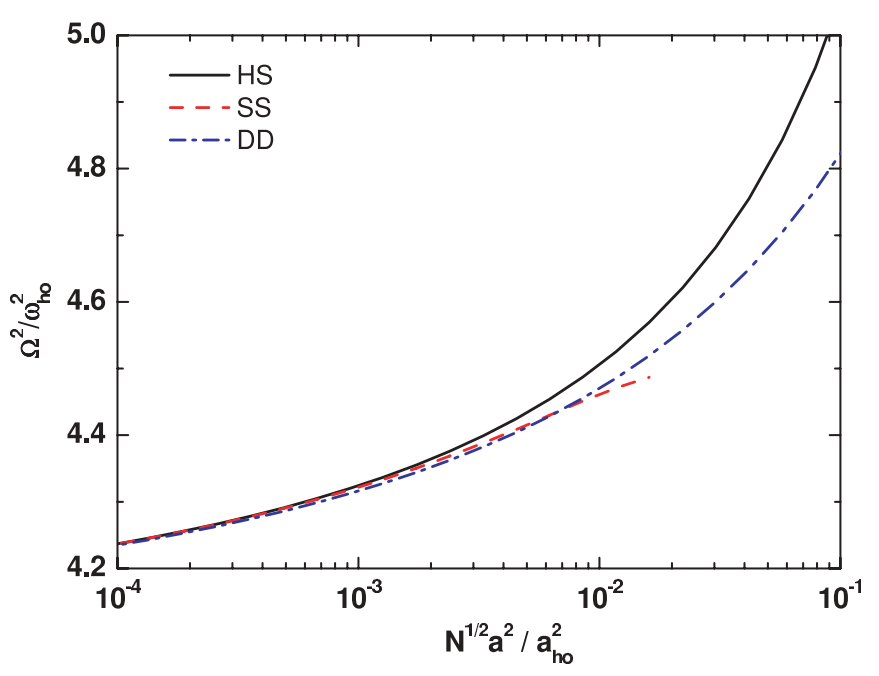

FIG. 4. (Color online) Comparison of the lowest breathing mode frequency $\Omega^{2}$ as a function of the coupling strength $N^{1 / 2} a^{2} / a_{\text {ho }}^{2}$ for different interaction potentials. The solid line corresponds to the hard disks and Eq. (16), the dashed line to the soft disks and Eq. (18), and the dash-dotted line to the dipoles and Eq. (16) with $a(k)$ as in Eq. (17).

The energetic properties of trapped gases can be accessed by observing the frequencies of collective oscillations. By displacing the center of the trap it is possible to generate oscillations that depend only on the frequency of the trapping potential. Instead, a sudden change in the frequency of the trap causes "breathing" oscillations, for which the frequency depends on the compressibility of the gas, which, in turn, is related to the equation of state. In Fig. 4 we show predictions for different interaction potentials in 2D systems. We use local density approximation and the equations of state deduced in the previous sections [see Eqs. (16)-(18)] to calculate the frequencies of the breathing mode. Out of all considered model interactions, the hard-core potential shows the strongest dependence. The soft-disk and dipolar potentials have softer dependencies.

\section{CONCLUSIONS}

To conclude, we have studied the energetic properties of dilute low-dimensional interacting Bose gases at zero temperature. In the regime of ultralow densities the equation of state is described by only a single dimensionless parameter; namely the gas parameter $n a^{D}$. The universal equation of state in 3D and 1D systems dates back to the 1960s [3,4,14]. The BMF terms of the 2D equation of state were obtained recently in Refs. [21-23] and their correctness was verified in Monte Carlo calculations [24]. When the density is increased, the details of the interaction potential become important and deviations from the universal behavior are observed.

In this work we propose to use an energy-dependent $s$-wave scattering length to describe the nonuniversal behavior. This method permits the generation of nonuniversal terms in the equation of state. The advantage of the proposed approach is that it is sufficient to know the energy-dependence of the $s$-wave scattering length for this method to be applicable. 
This permits us to use it in low dimensional systems, where the nonuniversal terms in the equation of state are not well established. Our proposal is the substitution of the $s$-weave scattering length in the universal equation of state by the energy-dependent scattering length $a(k)$ defined in Sec. II. The momentum $k$ of the two-body scattering problem is connected with the density of the many-body problem by considering that the scattering energy $\hbar^{2} k^{2} / m$ equals the mean-field term $g n / 2$. We effectively correct the coupling constant in such a way that the description is improved on the two-body level, thus adding nonuniversal corrections to the mean-field energy which provides the leading contribution to the energy. Although this seems to us a meaningful reasoning, it cannot be seen as a rigorous mathematical proof. In order to verify the proposed method we test this approach on 1D systems, where a direct comparison to exactly solvable models is done, and on 2D systems, where numerical results for different interaction potentials (hard disks, soft disks, dipoles) are used. The proposed approach works well for the problems we have studied and we hope it might be useful in other systems. We find that the typical density at which the non-universal terms become important is correctly estimated. For 1D systems, in the cases when the energy expansion can be obtained exactly, we show that the structure of the potential-dependent terms is predicted correctly. Finally we point out that nonuniversal terms can be studied experimentally by observing frequencies of collective oscillations.

\section{ACKNOWLEDGMENTS}

The work was partially supported by (Spain) Grant No. FIS2008-04403, Generalitat de Catalunya Grant No. 2005SGR-00779, and RFBR. G.E.A. acknowledges a post doctoral fellowship by MEC (Spain).

\section{APPENDIX A: EXACT EQUATION OF STATE OF WEAKLY-INTERACTING ONE-DIMENSIONAL BOSE GAS WITH $\delta$-PSEUDOPOTENTIAL INTERACTIONS}

The ground-state energy of a Lieb-Liniger gas can be found exactly using the Bethe ansatz approach. The energy as a function of the gas parameter is obtained implicitly by solving the following system of integral equations [4]:

$$
\begin{gathered}
e(\gamma)=\frac{\gamma^{3}}{\lambda^{3}} \int_{-1}^{1} k^{2} \rho(k) d k, \\
\gamma=\lambda / \int_{-1}^{1} \rho(k) d k, \\
\rho(k)=\frac{1}{2 \pi}+\int_{-1}^{1} \frac{2 \lambda \rho(\varkappa)}{\lambda^{2}+(k-\varkappa)^{2}} \frac{d \varkappa}{2 \pi},
\end{gathered}
$$

where $\gamma=-2 / n_{1 \mathrm{D}} a_{1 \mathrm{D}}$.

It is possible to obtain explicit expressions for the energy in terms of the gas parameter in the limits of small and large gas parameter as a series expansion. We solve the system of Eqs. (A1)-(A3) iteratively in the regime $|n a| \ll 1$. This is done by starting from $\rho^{(0)}=1 /(2 \pi)$ and substituting it into the right-hand side of Eq. (A3) to get $\rho^{(1)}$. The obtained expression is used for the next iteration and so on.
We provide an explicit expression for the ground-state energy close to the Tonks-Girardeau regime:

$$
\frac{E}{N}=\frac{\pi^{2} \hbar^{2} n^{2}}{6 m}\left[\sum_{l=0}^{\infty}(l+1)\left(-\frac{2}{\gamma}\right)^{l}+\frac{32 \pi^{2}}{15 \gamma^{3}}+O\left(\gamma^{-4}\right)\right] \text {. }
$$

The first two terms $\left[E / N=\frac{\pi^{2} \hbar^{2} n^{2}}{6 m}(1-4 / \gamma)\right]$ were obtained in the original work of Lieb and Liniger [4] (see also [61,62]).

We also rewrite Eq. (A4) in terms of the gas parameter $\tilde{n}=n_{1 \mathrm{D}} a_{1 \mathrm{D}}$ as

$$
\frac{E}{N}=\frac{\pi^{2} \hbar^{2} n^{2}}{6 m}\left[\sum_{l=0}^{\infty}(l+1) \tilde{n}^{l}-\frac{4 \pi^{2} \tilde{n}^{3}}{15}+O\left(\tilde{n}^{4}\right)\right] .
$$

The "excluded volume" contribution is intentionally separated from the nonuniversal part.

\section{APPENDIX B: EQUATION OF STATE OF A TWO-DIMENSIONAL BOSE GAS FROM CHERNY AND SHANENKO THEORY}

We note that the derivation of the equation of state of a weakly interacting Bose gas, proposed by Cherny and Shanenko [21], can be used to obtain an explicit expression of the energy as a function of the density $n$ :

$$
\begin{gathered}
\frac{E}{N}=\frac{2 \pi \hbar^{2} n(u)}{m}\left[u+\frac{u^{2}}{2}+2 u^{2} e^{2 / u} \operatorname{Ei}\left(-\frac{2}{u}\right)\right], \\
n(u) a^{2}=\frac{\exp (-2 \gamma-1 / u)}{\pi u},
\end{gathered}
$$

where $u$ (dimensionless in-medium scattering amplitude) defines the parametrical dependence of the energy on the density, $\operatorname{Ei}(x)=-\int_{x}^{\infty}\left(e^{-t} / t\right) d t$ is exponential integral function, and $\gamma$ is Euler's constant.

\section{APPENDIX C: FINITE-ENERGY SCATTERING PROBLEM FOR A SOFT-DISK POTENTIAL IN 2D}

In order to find the 2D $s$-wave scattering length of the soft-disk potential in $2 \mathrm{D}$ we have to solve the two-body scattering problem. The positive-energy Scrödinger equation for two particles of equal mass reads

$$
-\frac{\hbar^{2}}{m} \Delta f(r)+V(r) f(r)=\frac{\hbar^{2} k^{2}}{m} f(r),
$$

where $k$ is the relative momentum. We consider the soft-disk interaction potential and look for a spherically symmetric solution. The interaction potential is defined by the range of the potential $R_{0}$ and the height of the soft disk by

$$
V(r)=\left\{\begin{array}{cl}
\hbar^{2} \varkappa^{2} / m, & |r| \leqslant R_{0} \\
0, & |r|>R_{0} .
\end{array}\right.
$$

In the inner region, $r<R_{0}$, we use a solution that is regular at the origin,

$$
f(r)=I_{0}\left(r \sqrt{\varkappa^{2}-k^{2}}\right), \quad|r|<R_{0},
$$

where $I_{0}(x)$ is the modified Bessel function of the first kind. The normalization constant is not important for the present 
considerations. In the outer region the solution is simply a 2D plane wave

$$
f(r)=C_{1} J_{0}(k r)+C_{2} Y_{0}(k r), \quad|r|>R_{0},
$$

where $J_{0}(x)$ and $Y_{0}(x)$ are Bessel functions of the first and second kind, respectively. The coefficients $C_{1}$ and $C_{2}$ are obtained from the continuity condition for $f(r)$ and $f^{\prime}(r)$ at the edge of the soft disk $r=R_{0}$. This gives the following solution in the outer region, $r>R_{0}$.

$$
\begin{aligned}
f(r)= & \frac{\pi R_{0}}{2}\left\{\kappa I_{1}\left(\kappa R_{0}\right)\left[J_{0}\left(k R_{0}\right) Y_{0}(k r)-J_{0}(k r) Y_{0}\left(k R_{0}\right)\right]\right. \\
& \left.+k I_{0}\left(\kappa R_{0}\right)\left[J_{1}\left(k R_{0}\right) Y_{0}(k r)-J_{0}(k r) Y_{1}\left(k R_{0}\right)\right]\right\}
\end{aligned}
$$

where $\kappa=\left(\varkappa^{2}-k^{2}\right)^{1 / 2}$. The $s$-wave scattering length is the node of Eq. (C5) closest to the origin. We will consider the case of low densities, so that the incident particles are slow; $k \ll \varkappa$. Then $f(r)$ can be expanded in powers of $k$ and one has

$$
\begin{aligned}
f(r)= & I_{0}\left(\varkappa R_{0}\right)+\varkappa R_{0} I_{1}\left(\varkappa R_{0}\right) \ln \left(r / R_{0}\right) \\
& +\frac{k^{2} R_{0}^{2}}{4}\left[\left(1-r^{2} / R_{0}^{2}\right) I_{0}\left(\varkappa R_{0}\right)\right.
\end{aligned}
$$

$$
\begin{aligned}
& -\frac{2+\varkappa^{2}\left(R_{0}^{2}-r^{2}\right)-\varkappa^{2}\left(r^{2}+R_{0}^{2}\right) \ln \left(R_{0} / r\right)}{\varkappa R_{0}} \\
& \left.\times I_{1}\left(\varkappa R_{0}\right)\right]+O\left(k^{4}\right) .
\end{aligned}
$$

The zero-energy $s$-wave scattering length is found by setting $k=0$ in Eq. (C6) and leads to

$$
a_{0}=\exp \left[-\frac{I_{0}\left(\varkappa R_{0}\right)}{\varkappa R_{0} I_{1}\left(\varkappa R_{0}\right)}\right] R_{0} .
$$

Furthermore, one can set $r \rightarrow a_{0}$ in the second line of Eq. (C6) and find a correction to the position of the node

$$
a(k)=a_{0}-\alpha k^{2} a_{0}^{3}+O\left(k^{4}\right),
$$

$$
\alpha=\frac{R_{0}}{4 \varkappa a_{0}^{2}} \frac{I_{0}\left(\varkappa R_{0}\right)+I_{2}\left(\varkappa R_{0}\right)}{I_{1}\left(\varkappa R_{0}\right)}-\frac{1}{4}\left(\frac{R_{0}^{2}}{a_{0}^{2}}-1\right) .
$$

In order to test the accuracy of Eq. (C9) we also find numerically the nodes of the finite-energy scattering function Eq. (C5). A comparison of the exact result for the $s$-wave scattering length $a(k)$ to the expansion Eq. (C9) is presented in Fig. 2. We see that at the densities of interest, the obtained expansion works very well.
[1] L. D. Landau and E. M. Lifshitz, Quantum Mechanics: NonRelativistic Theory, Course of Theoretical Physics, Vol. 3, 3rd ed. (Pergamon Press, Oxford, 1977).

[2] E. M. Lifshitz and L. P. Pitaevskii, Statistical Physics, Part 2 (Pergamon Press, Oxford, 1980).

[3] M. Girardeau, J. Math. Phys. (NY) 1, 516 (1960).

[4] E. H. Lieb and W. Liniger, Phys. Rev. 130, 1605 (1963).

[5] M. H. Anderson, J. R. Ensher, M. R. Matthews, C. E. Wieman, and E. A. Cornell, Science 269, 198 (1995).

[6] K. B. Davis, M.-O. Mewes, M. R. Andrews, N. J. van Druten, D. S. Durfee, D. M. Kurn, and W. Ketterle, Phys. Rev. Lett. 75, 3969 (1995).

[7] A. Görlitz et al., Phys. Rev. Lett. 87, 130402 (2001).

[8] F. Schreck, L. Khaykovich, K. L. Corwin, G. Ferrari, T. Bourdel, J. Cubizolles, and C. Salomon, Phys. Rev. Lett. 87, 080403 (2001).

[9] M. Greiner, I. Bloch, O. Mandel, T. W. Hänsch, and T. Esslinger, Phys. Rev. Lett. 87, 160405 (2001).

[10] H. Moritz, T. Stöferle, M. Kohl, and T. Esslinger, Phys. Rev. Lett. 91, 250402 (2003).

[11] B. Laburthe Tolra, K. M. O'Hara, J. H. Huckans, W. D. Phillips, S. L. Rolston, and J. V. Porto, Phys. Rev. Lett. 92, 190401 (2004).

[12] T. Stöferle, H. Moritz, C. Schori, M. Köhl, and T. Esslinger, Phys. Rev. Lett. 92, 130403 (2004).

[13] T. D. Lee and C. N. Yang, Phys. Rev. 105, 1119 (1957).

[14] T. D. Lee, K. Huang, and C. N. Yang, Phys. Rev. 106, 1135 (1957).

[15] S. T. Beliaev, Zh. Eksp. Teor. Fiz. 34, 433 (1958) [Sov. Phys. JETP 7, 299 (1958)].
[16] K. A. Brueckner and K. Sawada, Phys. Rev. 106, 1117 (1957).

[17] E. Braaten, H.-W. Hammer, and S. Hermans, Phys. Rev. A 63, 063609 (2001).

[18] A. Bulgac, Phys. Rev. Lett. 89, 050402 (2002).

[19] D. Blume and C. H. Greene, Phys. Rev. A 65, 043613 (2002).

[20] V. Bendkowsky, B. Butscher, J. Nipper, J. P. Shaffer, R. Löw, and T. Pfau, Nature 458, 1005 (2009).

[21] A. Y. Cherny and A. A. Shanenko, Phys. Rev. E 64, 027105 (2001).

[22] C. Mora and Y. Castin, Phys. Rev. A 67, 053615 (2003).

[23] L. Pricoupenko, Phys. Rev. A 70, 013601 (2004).

[24] G. E. Astrakharchik, J. Boronat, J. Casulleras, I. L. Kurbakov, and Yu. E. Lozovik, Phys. Rev. A 79, 051602(R) (2009).

[25] N. N. Bogoliubov, J. Phys. (Moscow) 11, 23 (1947); reprinted in The Many-Body Problem, edited by D. Pines (Benjamin, New York, 1961).

[26] B. Sutherland, J. Math. Phys 12, 246 (1971).

[27] P. C. Hohenberg, Phys. Rev. 158, 383 (1967).

[28] V. N. Popov, Functional Integrals in Quantum Field Theory and Statistical Physics (Reidel, Dordrecht, 1983).

[29] C. Mora and Y. Castin, Phys. Rev. A 67, 053615 (2003).

[30] G. E. Astrakharchik and S. Giorgini, Phys. Rev. A 68, 031602(R) (2003).

[31] G. E. Astrakharchik and S. Giorgini, J. Phys. B 39, S1 (2006).

[32] G. E. Astrakharchik, D. M. Gangardt, Y. E. Lozovik, and I. A. Sorokin, Phys. Rev. E 74, 021105 (2006).

[33] A. S. Arkhipov, G. E. Astrakharchik, A. V. Belikov, and Yu. E. Lozovik, JETP Lett. 82, 39 (2005). 
[34] F. Mazzanti, G. E. Astrakharchik, J. Boronat, and J. Casulleras, Phys. Rev. Lett. 100, 020401 (2008).

[35] F. Mazzanti, G. E. Astrakharchik, J. Boronat, and J. Casulleras, Phys. Rev. A 77, 043632 (2008).

[36] It is worth mentioning that the beyond mean-field terms in threedimensional systems were first obtained for a hard-sphere gas by Lee, Huang, Yang $[13,14]$ and afterwards were shown to be universal $[4,15,16]$.

[37] G. E. Astrakharchik, J. Boronat, J. Casulleras, and S. Giorgini, Phys. Rev. Lett. 95, 190407 (2005).

[38] M. Gaudin, Phys. Lett. A24, 55 (1967).

[39] C. N. Yang, Phys. Rev. Lett. 19, 1312 (1967).

[40] V. Y. Krivnov and A. A. Ovchinnikov, Zh. Eksp. Teor. Fiz. 67, 1568 (1974) [Sov. Phys. JETP 40, 781 (1975)].

[41] G. E. Astrakharchik, Phys. Rev. A 72, 063620 (2005).

[42] F. Mazzanti, A. Polls, and A. Fabrocini, Phys. Rev. A 71, 033615 (2005).

[43] G. E. Astrakharchik, J. Boronat, I. L. Kurbakov, and Yu. E. Lozovik, Phys. Rev. Lett. 98, 060405 (2007).

[44] G. E. Astrakharchik, J. Boronat, J. Casulleras, I. L. Kurbakov, and Y. E. Lozovik, Phys. Rev. A 75, 063630 (2007).

[45] G. E. Astrakharchik, J. Boronat, J. Casulleras, I. L. Kurbakov, and Yu. E. Lozovik, Recent Progress in Many-Body Theories (World Scientific), Vol. 11, p. 245.

[46] Yu. E. Lozovik, I. L. Kurbakov, G. E. Astrakharchik, J. Boronat, and M. Willander, Solid State Commun. 144, 399 (2007).
[47] Yu. E. Lozovik, I. L. Kurbakov, G. E. Astrakharchik, and M. Willander, JETP 106, 296 (2008).

[48] H. P. Buchler, E. Demler, M. Lukin, A. Micheli, N. Prokof'ev, G. Pupillo, and P. Zoller, Phys. Rev. Lett. 98, 060404 (2007).

[49] C. Mora, O. Parcollet, and X. Waintal, Phys. Rev. B 76, 064511 (2007).

[50] L. Xing, Phys. Rev. B 42, 8426 (1990).

[51] S. Pilati, J. Boronat, J. Casulleras, and S. Giorgini, Phys. Rev. A 71, 023605 (2005).

[52] C. Mora and Y. Castin, Phys. Rev. Lett. 102, 180404 (2009).

[53] M. Schick, Phys. Rev. A 3, 1067 (1971).

[54] E. H. Lieb, R. Seiringer, and J. Yngvason, Commun. Math. Phys. 224, 17 (2001).

[55] V. N. Popov, Theor. Math. Phys. 11, 565 (1972).

[56] Yu. E. Lozovik and V. I. Yudson, Physica A 93, 493 (1978).

[57] A. Altmeyer, S. Riedl, C. Kohstall, M. J. Wright, R. Geursen, M. Bartenstein, C. Chin, J. H. Denschlag, and R. Grimm, Phys. Rev. Lett. 98, 040401 (2007).

[58] M. Olshanii, Phys. Rev. Lett. 81, 938 (1998).

[59] D. S. Petrov, M. Holzmann, and G. V. Shlyapnikov, Phys. Rev. Lett. 84, 2551 (2000).

[60] G. E. Astrakharchik and S. Giorgini, Phys. Rev. A 66, 053614 (2002).

[61] F. D. M. Haldane, Phys. Rev. Lett. 47, 1840 (1981).

[62] J. Brand, J. Phys. B: At. Mol. Opt. Phys. 37, S287 (2004). 